\title{
Generation of polycyclic groups
}

\author{
Martin Kassabov* \& Nikolay Nikolov
}

November 21, 2018

To Dan Segal on occasion of his 60-th birthday

\begin{abstract}
We give a new and self-contained proof of a theorem of Linnell and Warhurst that $d(G)-d(\widehat{G}) \leq 1$ for virtually polycyclic groups $G$. We also give a simple sufficient condition for equality $d(G)=d(\widehat{G})$ when $G$ is virtually abelian.
\end{abstract}

\section{Introduction}

Let $G$ be a finitely generated residually finite group. By $d(G)$ we denote the minimal size of a generating set for $G$, and by $d(\widehat{G})$ the minimal size of a generating set for the profinite completion $\widehat{G}$ of $G$. In other words

$$
d(\widehat{G})=\max \{d(G / N) \mid N \triangleleft G, G / N<\infty\} .
$$

Polycyclic groups are one of the best understood class of groups. For example most of the decision problems are decidable in this class, see [7].

It seems surprising therefore that it is still an open problem whether there exists an algorithm which finds $d(G)$ for any polycyclic group $G$ (given by say a set of generators and relations). This is unknown even in the case when $G$ is virtually abelian.

It is obvious that $d(G) \geq d(\widehat{G})$ and when there is equality both the value of $d(G)$ and a minimal generating set for $G$ can indeed be found algorithmically. (Say by enumerating both the finite images and all possibilities for generating sets for $G)$.

In general $d(G)-d(\widehat{G})$ can be arbitrarily large even for metabelian groups $G$, see [4. In fact Wise 9 has proved that there exist groups $G$ with arbitrarily large $d(G)$ while $d(\widehat{G})=3$.

Fortunately for polycyclic groups the situation is not that bad. In [3] Linnell and Warhurst proved the following theorem using methods from commutative algebra and lattices over orders.

${ }^{*}$ The author was partially supported by AMS Centennial Fellowship and NSF grant DMS 0600244. 
Theorem 1. Let $G$ be a virtually polycyclic group. Then $d(G) \leq d(\widehat{G})+1$.

Note that the inequality is sharp even for virtually abelian groups: many examples with $d(G)=d(\widehat{G})+1$ are constructed in $[5]$.

In this note we give an alternative proof of Theorem 1 While not claiming anything new we believe that our argument is much simpler that the original one in $[3$. Moreover our result gives some sufficient condition when $d(G)=d(\widehat{G})$ which can be verified quite easily in the case when $G$ is virtually abelian.

Theorem 2. Let $G$ be a group with normal finitely generated abelian subgroup $U$ such that $G / U$ is finite. Let $d_{p}(G)=d\left(G / U^{p}\right)$ for any prime $p$. Then

$$
\alpha=d(\widehat{G}) \leq d(G) \leq k:=\max \{\alpha, \beta+1\} \leq d(\widehat{G})+1,
$$

where $\alpha=\max _{p} d_{p}(G)$ and $\beta=\min _{p} d_{p}(G)$.

Moreover for any integer $N \in \mathbb{N}$ there exists a generating set $S$ for $G$ of size $k$, such that the first $d(\widehat{G})$ elements generate a subgroup of index co-prime to $N$. The same result holds for finitely generated virtually nilpotent groups.

In particular if there are two primes $p$ and $q$ such that $d_{p}(G) \neq d_{q}(G)$ then $d(G)=d(\widehat{G})$.

Note that Theorem 2 easily implies a weaker version of Theorem 1, namely that $d(G) \leq d(\widehat{G})+2$, however obtaining the right bound $d(\widehat{G})+1$ is harder.

For that we need a general and somewhat technical result (Theorem 7 on lifting generators) proved in Section 1. The proofs of Theorems 2 and 1 are then immediate and are given in Section 2

\section{Notation}

For elements $a, b \in G$ in a group $G$ the commutator $[a, b]$ of $a$ and $b$ is $a b a^{-1} b^{-1}$.

\section{$1 \quad$ Lifting generators}

In this section we shall prove a general result which under certain condition produces a generating set of a group $G$ starting from a generating set of some quotient $G / V$ of $G$. The reason for stating it in such generality is because in the next section we shall apply it in two settings: when $G$ a virtually abelian group and then when $G$ is virtually metabelian.

First recall the following result by Gaschütz ([1]).

Theorem 3. Let $G$ be a finite group with a normal subgroup $N$. Let $d \geq$ $d(G)$ and let $a_{1}, \ldots a_{d}$ be any $d$ elements which generate $G \bmod N$, i.e., $G=$ $N\left\langle a_{1}, \ldots, a_{d}\right\rangle$. Then we can find elements $g_{i} \in a_{i} N(i=1,2, \ldots, d)$ such that $G=\left\langle g_{1}, \ldots, g_{d}\right\rangle$. 
Definition 4. Let $G$ be a group and $p$ be a prime. A normal subgroup $L$ of finite index in $G$ is $p$-good if for any subgroup $H \leq G$ with $H L=G$ we have that $[G: H]$ is finite and coprime to $p$.

It is not difficult to see that $p$-good subgroups exists for any virtually polycyclic group $G$, see Lemma 11 below.

Now let $G$ be a finitely generated group with an abelian normal subgroup $V$ of finite rank. Suppose that for every prime number $p$ we have chosen a $p$-good subgroup $G_{p}$ of $G$ such that $G_{p} \geq V^{p}$.

Definition 5. We say that $h_{1}, \ldots, h_{k}$ generate $G \bmod p$ if $\left\langle h_{1}, \ldots, h_{k}\right\rangle G_{p}=G$. Let $d_{p}(G)=d\left(G / G_{p}\right)$ denote the minimal size of a set of generators for $G \bmod$ $p$.

Definition 6. Let $w=w\left(x_{1}, \ldots, x_{n}\right)$ be a group word (element in the free group $F$ ). The Fox derivatives $\frac{\partial w}{\partial x_{i}}$ are elements in the group ring $\mathbb{Z}[F]$, which are defined by $\frac{\partial x_{j}}{\partial x_{i}}=\delta_{i j}$ and

$$
\frac{\partial u v}{\partial x_{i}}=\frac{\partial u}{\partial x_{i}}+u \frac{\partial v}{\partial x_{i}} .
$$

Let $G$ be a group and $V$ be a $G$-module. For any $n$-tuple $\underline{g}=\left(g_{1}, \ldots, g_{n}\right) \in G^{n}$ the Fox derivative $\frac{\partial w}{\partial x_{i}}$ naturally defines a map $\frac{\partial w}{\partial x_{i}}(\underline{g}): V \rightarrow V$.

An equivalent way to define this map is the following: Let $\Gamma$ be an extension of $G$ by the abelian group $V$ then

$$
\frac{\partial w}{\partial x_{i}}(\underline{g})(a)=w\left(\gamma_{1}, \ldots, \gamma_{i-1}, a \gamma_{i}, \gamma_{i+1}, \ldots, \gamma_{n}\right) \cdot w\left(\gamma_{1}, \ldots, \gamma_{n}\right)^{-1}
$$

for any lifts $\gamma_{i} \in \Gamma$ of $g_{i} \in G$.

Theorem 7. Let $\gamma=\left(\gamma_{1}, \ldots \gamma_{k}\right)$ be a set of elements in $G$ which generate $G / V$. Suppose that $w\left(x_{1}, \ldots, x_{k}\right)$ is a word such that $w(\underline{\gamma}) \in V$.

Assume that

1. The image of the map $\pi: V \rightarrow V$ defined by $\pi(v)=\frac{\partial w}{\partial x_{k}}(\underline{\gamma}) \circ v$ has finite index $M$ in $V$.

2. For any choice of elements $g_{1} \in \gamma_{1} V, \ldots, g_{k-1} \in \gamma_{k-1} V$ the group $\left\langle g_{1}, \ldots, g_{k-1}\right\rangle$ generated by them has finite index in $G$.

3. We have that $d_{p}(G) \leq k$ for any prime $p$.

Then there exist lifts $g_{1}, \ldots, g_{k}$ of $\gamma_{1}, \ldots, \gamma_{k}$ (i.e. such that $g_{i} \in \gamma_{i} V$ ) which generate $G$.

Moreover there is an algorithm for finding $g_{i}$ from the $\gamma_{i}$ (provided all the objects from conditions 1,2,3 above are computable). 
Proof. We say that the element $g_{i}$ is a lift of $\gamma_{i}$ whenever $g_{i} \in \gamma_{i} V$. Note that the Fox derivative $\frac{\partial w}{\partial x_{i}}(\underline{g})=\frac{\partial w}{\partial x_{i}}(\underline{\gamma})$ does not depend on the choice of lifts $\underline{g}=\left(g_{1}, \ldots, g_{k}\right)$ of $\underline{\gamma}=\left(\gamma_{1}, \ldots, \gamma_{k}\right)$.

For each $i=0,1, \ldots, k$ let $Q(i)$ be the following statement.

$\mathbf{Q}(\mathbf{i})$ : There exist lifts $S_{i}=\left\{g_{1}, \ldots, g_{i}\right\}$ of $\gamma_{1}, \ldots, \gamma_{i}$ and a finite set of prime numbers $P_{i}$ with the following property.

- For each prime $p \in P_{i}$ there exist lifts $g_{j}^{(p)} \in \gamma_{j} V, j=i+1, \ldots, k$ such that $S_{i} \cup\left\{g_{j}^{(p)}\right\}_{j>i}$ are $k$ generators for $G \bmod p$.

- For each prime $p \notin P_{i}$, there exist lifts $g_{j}^{(p)}, j=i+1, \ldots, k-1$ such that for any lift $g_{k}^{(p)} \in \gamma_{k} V$ we have that $S_{i} \cup\left\{g_{j}^{(p)}\right\}_{j>i}$ are $k$ generators for $G$ $\bmod p$.

The proof of $Q(i)$ is by induction on $i$.

The base case $i=0$ is proved as follows: Take $S_{0}=\emptyset$ and choose any lifts $\gamma_{1}, \ldots, \gamma_{k-1}$ of the $\gamma_{j}$. They generate a subgroup of finite index $L$ in $G$, therefore for any $p \not L$ and any lift $g_{k} \in \gamma_{k} V$ the elements $\left\{g_{1}, \ldots, g_{k}\right\}$ generate $G \bmod p$.

Define $P_{0}$ to be the set consisting of all primes which divide $L$ or $M$. We only have to show that for all $p \in P_{0}$ there exist lifts $g_{1}^{(p)}, \ldots, g_{k}^{(p)}$, which generate $G \bmod p$. Consider the $k$ images $\bar{\gamma}_{j}$ of $\gamma_{1}, \ldots, \gamma_{k}$ in $G / V G_{p}$. They generate $G / V G_{p}$ (since $\gamma_{j}$ generate $\left.G / V\right)$ and also we know that $G / G_{p}$ is $k$-generated. By Gaschutz Theorem we can find elements $g_{j}^{(p)} \in \gamma_{j} V G_{p}$ which generate $G / G_{p}$. These can be further adjusted by elements from $G_{p}$ so that $g_{j}^{(p)} \gamma_{j}^{-1} \in V$. This proves the base case $i=0$ of the induction.

Suppose that we have already found $S_{i}$ and $P_{i}$. By the Chinese Remainder Theorem there exists a lift $g_{i+1}$ such that $g_{i+1}=g_{i+1}^{(p)}\left(\bmod G_{p}\right)$ for all $p \in P_{i}$.

Choose any lifts $g_{i+2}, \ldots g_{k-1}$. By one of the assumptions the group $H$ generated by $\left\{g_{i}\right\}_{i=1}^{k-1}$ is of finite index $N_{i}$ in $G$. Denote $P_{i+1}=\left\{p \mid p\right.$ divides $\left.N_{i}\right\} \cup P_{i}$.

We want to show that $S_{i+1}=S_{i} \cup\left\{g_{i+1}\right\}$ and the set $P_{i+1}$ satisfy the induction hypothesis.

It is very easy to check that the second part of the induction hypothesis is satisfied for this definition of the set $P_{i+1}$ (just choose $g_{j}^{(p)}=g_{j}$ for $j=$ $i+2, \ldots, k-1)$. It remains to show that for all primes $p \in P_{i+1}$ the first condition is satisfied. This is clearly the case if $p \in P_{i}$. Let $p \notin P_{i}$ then by the induction assumption there exist lifts $\left\{g_{j}^{(p)}\right\}_{j=i}^{k-1}$ which together with $S_{i}$ and any lift $g_{k}^{(p)} \in \gamma_{k} V$ generate $G \bmod p$. We will show that we can chose a lift $g_{k}$ of $\gamma_{k}$ such that the group $L$ generated by $S_{i+1},\left\{g_{j}^{(p)}\right\}_{j=i+1}^{k-1}$ and $g_{k}$ contains an element $u \equiv g_{i+1}^{-1} g_{i+1}^{(p)}\left(\bmod V^{p}\right)$, which implies that these elements generate $G$ $\bmod p$. 
The key observation here is that for $x \in V$ the element $w\left(g_{1}^{(p)}, \ldots, g_{k-1}^{(p)}, x g_{k}^{(p)}\right)$ is equal to

$$
\left(\frac{\partial w}{\partial x_{k}}(\underline{\gamma}) \cdot x\right) w\left(g_{1}^{(p)}, \ldots, g_{k-1}^{(p)}, g_{k}^{(p)}\right)=\pi^{\prime}(x) \in V
$$

which by one of the assumptions takes any value in $V / V^{p}$ as $x$ ranges over $V / V^{p}$ (since $p$ does not divide the index $M$ of the image of $\frac{\partial w}{\partial x_{k}}(\underline{\gamma})$ in $V$ ). So we can indeed find $x \in V$ such that the element $\pi^{\prime}(x) \in V$ satisfies $\pi^{\prime}(x) \equiv g_{i+1}^{-1} g_{i+1}^{(p)}$ $\bmod V^{p}$.

This shows that there exist lifts $g_{k}$ which generate $G \bmod p$ which completes the induction step.

The statement $Q(k)$ gives a set $S_{k}$ which generates $G \bmod p$ for any prime $p$ and therefore $\left\langle S_{k}\right\rangle=G$.

It is clear that this argument in fact produces an algorithm for finding the set $S_{k}$ in a very efficient way, of course provided the various subgroup indices, words and maps $G \rightarrow G / G_{p}$ involved in the induction are computable. Theorem 7 is proved.

Remark 8. A slight modification of the proof gives that for any finite set $P$ of primes such that $d_{p}(G)<k$, we can find lifts $g_{1}, \ldots, g_{k-1}$ which together with any lift of $\gamma_{k}$ generate a subgroup of index not divisible by any prime in $P$.

Remark 9. If $\gamma_{k}=e$ then we can take the word $w=x_{k}$. Its Fox derivative is $\frac{\partial w}{\partial x_{k}}=e$ and defines the identity map from $V$ to $V$, which is clearly surjective.

Remark 10. If we replace the assumption that $V$ is abelian with $V$ nilpotent, then all results remain valid, since a set generates a nilpotent group if and only if it generates the abelianization of the group.

\section{Applications of Theorem 7}

\subsection{Proof of Theorem 2}

Proof. Clearly $d(\widehat{G})=\alpha$. Let $k:=\max \{\beta+1, \alpha\}$.

Take $V:=U^{q}$ where $q$ is a prime such that $\beta=d_{q}(G)$. Then $V$ is $q$-good, i.e., any collection of elements which generates $G / V$ generates a subgroup of finite index (coprime to $q$ ) in $G$.

Take elements $\gamma_{1}, \ldots, \gamma_{\beta}$ which generate $G / V$. Set $\gamma_{i}=1$ for $j=\beta+1, \ldots, k$. It is easy to see that the group $G$, subgroups $V, G_{p}=V^{p}$ (for any prime $p$ ), the elements $\gamma_{i}$ above, and the word $w=x_{k}$ satisfy the conditions of Theorem 7 We conclude that $G$ can be generated by some lifts of $\gamma_{1}, \ldots, \gamma_{k}$ and so $d(G) \leq k$ as claimed.

For the second part of the theorem start with $V=U^{N}$ instead and with any generating set $\gamma_{1}, \ldots, \gamma_{s}$ for $G / V$ and again take $\gamma_{j}=1, s<j \leq k$. The rest of the argument is similar. 


\subsection{Proof of Theorem 1}

We begin with the following straightforward

Lemma 11. If $G$ is a virtually polycyclic group and $p$ is a prime then $G$ has a p-good subgroup $L$.

Proof. We use induction on the Hirsch length $h(G)$ of $G$. When $h(G)=0$ then $G$ is finite and we can simply take $L=1$. Suppose that the Lemma has been proved for all groups of Hirsch length less than $h>0$. Consider a virtually polycyclic group $G$ with $h(G)=h$. Since $G$ is infinite it has an infinite normal abelian subgroup $N$. Let $\bar{G}=G / N^{p}$. Clearly $h(\bar{G})<h$ and so $\bar{G}$ has a $p$-good subgroup, say $\bar{L}=L / N^{p}$ for some normal subgroup $L$ containing $N^{p}$.

Then $L$ is $p$-good for $G$ : Suppose $H \leq G$ with $H L=G$. Then $\overline{H L}=\bar{G}$ where $\bar{H}=H N^{p} / N^{p}$ and so the index $\left[G: H N^{p}\right]$ is finite and coprime to $p$. Therefore $H N=H N^{p}$ (since $N / N^{p}$ is a power of $p$ while $\left[H N: H N^{p}\right]$ must be coprime to $p$ ) and so $H_{1} N^{p}=N$ where $H_{1}=H \cap N$. The last equality implies that $\left[N: H_{1}\right]$ is finite and coprime to $p$ and hence so is $[G: H]=[G: N H]\left[N: H_{1}\right]$.

Notice that the $p$-good subgroup $L$ we found contains $N^{p}$.

Lemma 11 raises the following natural

Problem 1. Which groups $G$ possess a subgroup $N$ of finite index such that $N H=G$ for a subgroup $H<G$ implies that $[G: H]<\infty$ ?

The purpose of the following three Lemmas is to ensure the existence of suitable word $w$, subgroup $V$ and elements $g_{1}, \ldots g_{k}$ in a virtually metabelian and polycyclic group $G$ which meet the conditions of Theorem 7

Lemma 12. Let $G$ be a virtually metabelian and polycyclic group. Then there exist normal subgroups $G_{0} \triangleright V$ of $G$ such that

1. $G / G_{0}$ is finite, $V$ is a torsion free abelian group,

2. if $H$ is a subgroup of $G$ such that $H G_{0}=G$ then $H$ is of finite index in $G$,

3. $G_{0} / V$ is a nilpotent group which acts commutatively on $V$, i.e., $G_{0} / C_{G_{0}}(V)$ is abelian, and

4. $\mathbb{Q} \otimes V$ is a perfect $\mathbb{Q}\left[G_{0} / V\right]$ module, i.e., $\left(G_{0}-1\right) \cdot V$ has finite index in $V$.

Proof. Let $A<B$ be normal subgroups of $G$ such that $A$ and $B / A$ are torsion free abelian and $[G: B]$ is finite. Let $L$ be a $p$-good subgroup of $G$ for some prime $p$ and take $G_{0}=B \cap L$. Then $G_{0}$ also a $p$-good subgroup of $G$ and item 2 follows.

Let $W=A \cap G_{0}$. We have that $W$ and $G_{0} / W$ are torsion free abelian groups and $W$ is a module for $\bar{G}_{0}=G_{0} / W$. Consider the chain of submodules $W \geq\left(G_{0}-1\right) W \geq\left(G_{0}-1\right)^{2} W \geq \cdots$. This is a chain of subgroups of the finitely generated abelian group $W$, so let $V$ be the first module in that series 
such that $\left(G_{0}-1\right) V$ has finite index in $V$. Clearly $G_{0} / V$ is a nilpotent group (since $G_{0}$ acts nilpotently on $\left.W / V\right)$. Item 4 is clear since $\left[V:\left(G_{0}-1\right) V\right]$ is finite while item 3 follows since $C_{G_{0}}(V) \geq W$ and $G_{0} / W$ is abelian.

Lemma 13. Let $\Gamma_{0}$ be a finitely generated torsion free abelian group and let $V$ be a finitely generated torsion free $\mathbb{Z}\left[\Gamma_{0}\right]$ module such that $V_{\mathbb{Q}}=V \otimes \mathbb{Q}$ is a perfect $\mathbb{Q}\left[\Gamma_{0}\right]$-module. Then there exists an integer $N$ such that for any subgroup $\Gamma<\Gamma_{0}$ of index co-prime to $N$ we have that $V_{\mathbb{Q}}$ is a perfect $\mathbb{Q}[\Gamma]$-module.

Further when this happens then we can find an integer $M \in \mathbb{N}$ (depending on $\Gamma)$, integers $s_{i}$ and group elements $h_{i} \in \Gamma$ such that

$$
\sum s_{i}\left(1-h_{i}\right)=M \cdot i d
$$

as operators on $V$.

Proof. Let $\chi$ be a irreducible character (over $\mathbb{C}$ ) of $\Gamma_{0}$. We will call $\chi$ a character of finite order if all values of $\chi$ are roots of 1 , in this case the order of $\chi$ is the least integer $n$ such that all its values are $n$-th roots of 1 .

Since $V$ is a perfect $\mathbb{Q}\left[\Gamma_{0}\right]$ module it does not contain a trivial submodule. Let $N$ be the gcd of all orders of irreducible characters which appears in $\mathbb{C} V$. If $\Gamma \leq \Gamma_{0}$ is a subgroup of index co-prime to $N$ then the restriction of any irreducible characters in $V$ to $\Gamma$ is non trivial. Therefore $\mathbb{C} V$ is a perfect $\mathbb{C}[\Gamma]$ module, which implies that $V_{\mathbb{Q}}=\mathbb{Q} \otimes V$ is perfect $\mathbb{Q}[\Gamma]$ module.

For the second part, let $I$ be the augmentation ideal of $\mathbb{Q} \Gamma$. Since $V_{\mathbb{Q}}=I V_{\mathbb{Q}}$ and $V_{\mathbb{Q}}$ is a finite dimensional vector space over $\mathbb{Q}$ we have by Nakayama's lemma that $i d+T$ annihilates $V_{\mathbb{Q}}$ for some $T \in I$.

Expressing $T$ in the basis of $I$ and clearing the common denominator $M$ of the rational coefficients gives the integers $s_{i}$ and the elements $h_{i} \in G$.

Lemma 14. Let $\Gamma$ be a group with an abelian normal subgroup $V$ such that $\left[\Gamma^{\prime}, V\right]=1$ and $\Gamma / V$ is nilpotent of class d. Let $g_{1}, \ldots, g_{k-1} \in \Gamma$.

For any integers $t \in \mathbb{N}, s_{i}$ and group elements $h_{i} \in\left\langle g_{1}, \ldots, g_{k-1}\right\rangle \quad(i=$ $1, \ldots, t)$ there exists a word $w$ on $x_{1}, \ldots, x_{k}$ such that

- $w\left(g_{1}, \ldots, g_{k-1}, g\right) \in V$ for any $g \in \Gamma$

- The action of Fox derivative $\frac{\partial w}{\partial x_{k}}\left(g_{1}, \ldots, g_{k-1}, g\right)$ on $x \in V$ is given by

$$
x \mapsto\left(\sum_{i=1}^{t} s_{i}\left(1-h_{i}\right)\right)^{d} \cdot x .
$$

Proof. Consider the word $w^{\prime}\left(g_{1}, \ldots, g_{k-1}, g\right)=\prod\left[g, h_{i}\right]^{s_{i}}$ where $h_{i}$ are expressed as words on $g_{1}, \ldots, g_{k-1}$. A direct computation gives that the Fox derivative $\frac{\partial w}{\partial x_{k}}$ at $\left(g_{1}, \ldots, g_{k-1}, g\right)$ with respect to the last variable acts on $V$ as multiplication by $\sum s_{i}\left(1-h_{i}\right) \in \mathbb{Z}[\Gamma / V]$ (use that $\left[a g, h_{i}\right]=a\left[g, h_{i}\right]\left({ }^{h_{i}} a\right)^{-1}={ }^{\left(1-h_{i}\right)} a \cdot\left[g, h_{i}\right]$ and each $\left[g, h_{i}\right]$ acts trivially on $\left.V\right)$. 
Iterating the map $g \rightarrow w^{\prime}\left(g_{1}, \ldots, g_{k-1}, g\right) \quad d$ times gives a word $w$ :

$w\left(x_{1}, \ldots, x_{k}\right)=w^{\prime}\left(x_{1}, \ldots, x_{k-1}, w^{\prime}\left(x_{1}, \ldots, x_{k-1}, w\left(\cdots w^{\prime}\left(x_{1}, \ldots, x_{k-1}, x_{k}\right) \ldots\right)\right)\right.$.

The Fox derivative of $w$ with respect to $x_{k}$ is $\left(\sum s_{i}\left(1-h_{i}\right)\right)^{d}$, because substitution of words corresponds to multiplication of Fox derivatives. The word $w$ always evaluates to one on $\Gamma / V$, because the group $\Gamma / V$ is nilpotent of class $d$.

We now have all the ingredients to prove Theorem 1 It will follow from the corresponding result for metabelian groups:

Theorem 15. Let $G$ be a virtually metabelian polycyclic group. Then

$$
d(\widehat{G}) \leq d(G) \leq d(\widehat{G})+1 .
$$

Proof. Let $k=d(\widehat{G})+1$. Let $G_{0}$ and $V$ are the subgroups provided by Lemma 12. Now Lemma 13 applied to the group $G_{0} /\left[G_{0}, G_{0}\right] V$ acting on $V$, gives us an integer $N$ such than any subgroup of index co-prime to $N$ in $G_{0} / V$ acts perfectly on $V$ (as a rational module).

By Lemma 2 there exists a generating set $S=\left\{\gamma_{1}, \ldots, \gamma_{k}\right\}$ of $G / V$ such that $\gamma_{k} \in G_{0}$ and the subgroup $\Gamma=\left\langle\gamma_{1}, \ldots, \gamma_{k-1}\right\rangle V \cap G_{0}$ has index $\left[G_{0}: \Gamma\right]$ co-prime to $N$. Therefore $V$ is a perfect rational $\mathbb{Q}[\Gamma / V]$-module and for some integer $M$ and element $T$ in the augmentation ideal of $\mathbb{Z}[\Gamma / V]$ we have that $T$ acts on $V$ as multiplication by $M$.

For each prime $p$ pick a $p$-good subgroup $G_{p}$ of $G$ containing $V^{p}$. (In fact by replacing $G_{p}$ with a normal subgroup of $G$ of finite index we may even assume $G_{p} \cap V=V^{p}$.)

Now apply Lemma 14 to $\Gamma$ and $V$ with $\gamma_{i}=g_{i},(i=1, \ldots, k-1), g=\gamma_{k}$ and $s_{i} \in \mathbb{N}, h_{i} \in\left\langle\gamma_{1}, \ldots, \gamma_{k-1}\right\rangle$ chosen so that $T \equiv \sum_{i} s_{i}\left(1-h_{i}\right)$ in $\mathbb{Z}[\Gamma / V]$. We conclude that there is a word $w\left(x_{1}, \ldots, x_{k}\right)$ such that $w(\underline{\gamma}) \in V$ and $\frac{\partial w}{\partial x_{k}}(\underline{\gamma})$ acts on $V$ as multiplication by $M^{d}$ where $d$ is the nilpotency class of $\Gamma / V$.

We can now apply Theorem 7 to $G$ with these choices of $w, V, G_{p}$ and $\gamma_{j}$. The conditions 1,2 and 3 are satisfied by the construction of the subgroups $G_{0}$ and $V$, the word $w$ and the definition of the number $k$. So by Theorem 7 we can find lifts $a_{i} \in \gamma_{i} V$ such that $G=\left\langle a_{1}, \ldots, a_{k}\right\rangle$. Theorem [15] is proved.

Remark 16. If we have that $d(G / V)<d(\widehat{G})$ then the argument above gives that $d(G)=d(\widehat{G})$.

Proof of Theorem 11. In general a virtually polycyclic group $G$ is virtually nilpotent by abelian, i.e., it has normal subgroups $G_{1}>G_{2}$ such that $G / G_{1}$ is finite, $G_{1} / G_{2}$ is abelian while $G_{2}$ is nilpotent. (See Theorem 2, Chapter 2 in 6 ).

Now every group which generates $H=G / G_{2}^{\prime}$ generates $G$ and so we have $d(G)=d(H), d(\widehat{G})=d(\widehat{H})$. Thus Theorem 1 becomes a corollary of Theorem 
[15. Moreover its proof gives an efficient algorithm for generating a polycyclic group $G$ with $d(\widehat{G})+1$ elements, even with $d(\widehat{G})$ elements if the condition of Remark [16 holds.

\section{Acknowledgement}

We thank B. Petrenko for his preprint [2, which contains an argument on the generation of rings which led us to Theorem 7 . Similar result for modules can be found in 8 . The first author thanks Imperial College, London for the hospitality during his visit.

\section{References}

[1] W. Gaschütz, Zu einem von B.H und H. Neumann gestellen Problem, Math. Nachr. 14 (1956), 249-252.

[2] R.V. Kravchenko, B.V. Petrenko, Some formulas for the smallest number of generators for finite direct sums of matrix algebras. arXiv: math/0611674v7.

[3] P.A. Linnell, D. Warhurst, Bounding the number of generators of a polycyclic group, Arch math. Vol 37 (1981) 7-17,

[4] G.A. Noskov. Number of generators of a group, Mat. Zametki 33, no. 4 (1981), 489-498.

[5] G.A. Noskov, On the number of generators of crystallographic groups, Sibirsk. Mat. Zh. 30 (1989), no. 2, 145-150; translation in Siberian Math. J. 30 (1989), no. 2, 284-289.

[6] D. Segal, Polycyclic groups, Cambridge University Press, 1983.

[7] D. Segal, Decidable properties of polycyclic groups. Proc. London Math. Soc. (3) 61 (1990), no. 3, 497-528.

[8] R.G. Swan, The number of generators of a module, Math. Z. 1021967 $318-322$.

[9] D. Wise, A residually finite version of Rips's construction. Bull. London Math. Soc. 35 (2003), no. 1, 23-29. 